\title{
Left Ventricular Hypertrophy: Major Risk Factor in Patients with Hypertension: Update and Practical Clinical Applications
}

\author{
Richard E. Katholi and Daniel M. Couri \\ Prairie Cardiovascular Consultants, Ltd., 619 E. Mason Street, Ste. 4P57, Springfield, IL 62701, USA \\ Correspondence should be addressed to Richard E. Katholi, rkatholi@prairieheart.com
}

Received 15 February 2011; Accepted 19 April 2011

Academic Editor: Amgad N. Makaryus

Copyright ( $) 2011$ R. E. Katholi and D. M. Couri. This is an open access article distributed under the Creative Commons Attribution License, which permits unrestricted use, distribution, and reproduction in any medium, provided the original work is properly cited.

\begin{abstract}
Left ventricular hypertrophy is a maladaptive response to chronic pressure overload and an important risk factor for atrial fibrillation, diastolic heart failure, systolic heart failure, and sudden death in patients with hypertension. Since not all patients with hypertension develop left ventricular hypertrophy, there are clinical findings that should be kept in mind that may alert the physician to the presence of left ventricular hypertrophy so a more definitive evaluation can be performed using an echocardiogram or cardiovascular magnetic resonance. Controlling arterial pressure, sodium restriction, and weight loss independently facilitate the regression of left ventricular hypertrophy. Choice of antihypertensive agents may be important when treating a patient with hypertensive left ventricular hypertrophy. Angiotensin-converting enzyme inhibitors or angiotensin II receptor blockers followed by calcium channel antagonists most rapidly facilitate the regression of left ventricular hypertrophy. With the regression of left ventricular hypertrophy, diastolic function and coronary flow reserve usually improve, and cardiovascular risk decreases.
\end{abstract}

\section{Introduction}

While there are many proven benefits for the successful longterm treatment of hypertension including decreasing risk of stroke, renal failure, and myocardial infarction, an increasingly important area of hypertension treatment is to identify left ventricular hypertrophy and prescribe a combination of therapies which facilitates regression to improve patients' symptoms and prognosis. Left ventricular hypertrophy is both a major maladaptive response to chronic pressure overload and an important risk factor in patients with hypertension. The development of left ventricular hypertrophy is highly correlated with systolic hypertension. In the Framingham Heart Study, even borderline isolated systolic hypertension at an elderly age was associated with increased left ventricular wall thickness and impaired diastolic filling [1]. Complications of left ventricular hypertrophy include atrial fibrillation, diastolic heart failure, systolic heart failure, and sudden death. Both earlier recognition and improved understanding of cardiac hypertrophy may lead to more effective therapeutic strategies for this cardiovascular risk factor. This paper will focus on concentric left ventricular hypertrophy and discuss differential diagnosis, which techniques are available to identify left ventricular hypertrophy, and therapies to decrease patients' risks and to facilitate regression to improve symptoms and prognosis. This paper will also discuss novel therapies in development which may have a significant impact on this high-risk problem in the future.

\section{Factors Promoting Left Ventricular Hypertrophy}

It is now appreciated that left ventricular hypertrophy is mediated not only by the mechanical stress of pressure overload, but also by various neurohormonal substances that independently exert trophic effects on myocytes and nonmyocytes in the heart [2]. As shown in Table 1, trophic factors include angiotensin II, aldosterone, norepinephrine, and insulin which directly promote myocyte hypertrophy and matrix deposition independent of their effects on systemic arterial pressure $[3,4]$. These trophins stimulate the production of a series of cytokines and growth factors 
TABLE 1: Factors promoting left ventricular hypertrophy.

Hypertension
Neurohumoral factors (growth stimulation)
Angiotensin II
Aldosterone
Norepinephrine
Insulin and other growth factors
Genetic influences

including transforming growth factor beta, fibroblast growth factor, and insulin growth factor that directly stimulate cardiac protein synthesis and hypertrophy. While elevated systemic arterial pressure plays a role in the pathogenesis of left ventricular hypertrophy, the extent of cardiac growth and response to increased pressure loading is not uniform among patients suggesting genetic mechanisms in cardiac hypertrophy [5]. Thus, patients with moderate arterial hypertension present with a wide range of left ventricular mass, ranging from normal heart to severe hypertrophy. In addition, the remodeling of the left ventricle may take a concentric or an eccentric form independent of the extent of blood pressure elevation.

\section{Clinical Suspicion for Left Ventricular Hypertrophy}

The primary care physician encounters many patients with hypertension. An electrocardiogram (but not an echocardiogram) is suggested in the initial evaluation of patients with hypertension to detect arrhythmias, evidence of ischemic heart disease, and/or left ventricular hypertrophy [6]. Since not all patients with hypertension develop left ventricular hypertrophy, there are clinical findings that should be kept in mind that may alert the physician to the presence of left ventricular hypertrophy so a more definitive evaluation can be performed. The detection of left ventricular hypertrophy is important because these patients' risk of cardiovascular morbidity and mortality is two-to-four-fold increased compared to patients with normal left ventricular mass $[7,8]$. Although echocardiography is a more sensitive tool for identifying left ventricular hypertrophy, cost has kept it from becoming part of the routine evaluation of an uncomplicated asymptomatic patient with hypertension. If an S4 gallop is noted on physical examination, one should consider early diastolic dysfunction and possible left ventricular hypertrophy. Abnormal electrocardiogram findings include left atrial enlargement, prolongation of the QT interval, and left ventricular hypertrophy. If graded exercise testing is done, it is often noticed that patients with left ventricular hypertrophy demonstrate a hyperadrenergic response with increases in heart rate to 150 beats per minute within the first minute and excessive elevations of systolic blood pressure of greater than $200 \mathrm{~mm} \mathrm{Hg}$. A hyperadrenergic response of heart rate and blood pressure explains the patient's dyspnea on exertion and is also thought to reflect the presence of left ventricular hypertrophy [9].
There are a number of distinguishing clinical features which appear to identify specific subgroups of hypertensive patients who have left ventricular hypertrophy. Of patients with left ventricular hypertrophy presenting with anginal equivalent symptoms, $85 \%$ of these patients have been found to be women with a mean age of 63 years, and $83 \%$ had exogenous obesity [10]. Thus, the typical hypertensive patient that one should be suspicious of having left ventricular hypertrophy is an overweight, middleaged, postmenopausal female who presents to a physician with a history of exertional chest pain that is typical of angina pectoris. The patient will often give a history of hypertension, but, typically, it is not severe. In addition, the patient commonly has no smoking history, is not glucose intolerant, and has a borderline dyslipidemia. These findings superimposed on a positive graded exercise test are suggestive of hypertensive heart disease in women. That they developed concentric left ventricular hypertrophy disproportionate to the arterial pressure and had associated obesity suggests a genetic basis for this problem. Left ventricular hypertrophy in women is now considered to be a strong cardiovascular risk factor independent of blood pressure [11, 12]. Studies reveal an increased incidence in atrial fibrillation and sudden death in women with left ventricular hypertrophy. In addition, hypertensive heart disease is a common cause of congestive heart failure in women. One can only speculate why postmenopausal women with mild-to-moderate left ventricular hypertrophy have more angina than men with a similar degree of left ventricular hypertrophy. Speculation includes that women have smaller coronary arteries and/or greater perception of pain because of the loss of analgesic effects of estrogen and/or because loss of estrogen results in less endothelial nitric oxide production in the coronary vascular tree $[13,14]$.

Metabolic syndrome has been associated with an increased left ventricular mass in recent reports. Even after controlling for age, sex, and 24-hour systolic blood pressure, subjects with metabolic syndrome have significantly greater left ventricular mass [15]. The lack of association between left ventricular mass and blood pressure in patients with metabolic syndrome suggests other factors such as insulin resistance and hyperinsulinemia, sodium retention, sympathetic activation, and adipose tissue peptides such as leptin may be responsible. Accordingly, the primary care physician should be aware of the impact of metabolic syndrome on left ventricular mass in overweight and obese hypertensive patients and screen this high-risk group for this cardiovascular complication. Other patient types that should be kept in mind so that the primary care physician is alert to pursue a workup for left ventricular hypertrophy is the obese patient who should be screened for sleep apnea. Patients who have sustained the elevation of blood pressure throughout the night and do not have the normal nocturnal fall of arterial pressure are at increased risk of developing left ventricular hypertrophy [16]. African American and Caribbean and Mexican-origin Hispanic patients with hypertension also have a higher incidence of left ventricular hypertrophy and, thus, the clinician should have a higher index of suspicion and consider screening for left ventricular hypertrophy [17]. 
Most studies suggest that African American and Caribbean and Mexican-origin Hispanic patients have not only a greater left ventricular wall thickness, but also a greater left ventricular mass, even with equivalent blood pressures than white patients. The most difficult patient to identify is the patient with "masked" hypertension. This subset of patients reacts in an opposite way to patients with "white coat" hypertension [18]. Instead of the patient's blood pressure being elevated when sitting in a doctor's office, the blood pressures are normal. However, when these patients are performing daily activities, including exercise, they have an excessive elevation in systolic blood pressure. Thus, throughout the day, their heart has increased afterload which acts as a stimulus for hypertrophy when they are undergoing normal activities. Arteriolar thickening on funduscopic examination, S4 gallop, abnormal EKG, or exercise testing may suggest that the patient has "masked" hypertension, and ambulatory blood pressure monitoring can confirm this subset of hypertensive patients.

Left ventricular hypertrophy should also be suspected in hypertensive patients found to have unilateral renal artery stenosis $[19,20]$. There is evidence that the activation of the renin-angiotensin-aldosterone system may contribute to the development of left ventricular hypertrophy independent on effects of blood pressure. Left ventricular hypertrophy has been shown to be three times more prevalent in patients with functionally significant unilateral renal artery stenosis compared to patients with essential hypertension.

There also appears to be an increase in left ventricular mass beyond the amount needed to compensate the blood pressure-related hemodynamic load in patients with primary aldosteronism [21]. Because hypertension and obesity increase with age, the prevalence of left ventricular hypertrophy may be 50 percent in patients over 65 years of age [22]. Finally, there is a high prevalence of left ventricular hypertrophy in patients with renal insufficiency. Left ventricular hypertrophy is present in approximately 70 percent of patients at the initiation of dialysis [23].

\section{Differential Diagnosis of Left Ventricular Hypertrophy and Imaging Approaches to Differentiate Physiologic from Pathologic Hypertrophy}

The adult myocyte is a terminally differentiated cell that does not divide in response to stress $[3,4]$. Thus, the myocyte adapts to a pressure overload by the replication of sarcomeres and by changes in enzyme isoforms which regulate contractile function and ion transport, resulting in a more efficient, energy-conserving contraction. Hypertrophy secondary to exercise or hyperthyroidism appears appropriate in regard to remodeling of the left ventricular with relative dilatation which represents advantageous use of Frank-Starling forces to achieve greater efficiency of energy use and because coronary flow reserve is appropriate for the degree of myocardial growth $[24,25]$. In contrast, left ventricular hypertrophy secondary to essential hypertension is pathological with stimulus to growth also activating fibroblasts leading to an interstitial fibrosis which is another feature of the structural remodeling of the myocardium found in hypertensive heart disease. Thus, in left ventricular hypertrophy secondary to essential hypertension, left ventricular chamber size is smaller with elevated diastolic filling resulting in a blunting of the heart's ability to efficiently use energy, and coronary flow reserve remains below that expected for the degree of hypertrophy [26].

If a patient has hypertension and also participates in a regular intensive aerobic activity such as jogging or bicycling, differentiating physiologic from pathologic increase in left ventricular mass is an important and challenging assessment. Chronic aerobic exercising can cause electrocardiographic changes that can mimic pathological conditions and highly conditioned individuals can harbor suspected cardiovascular disease despite the absence of cardiac symptoms [27]. Distinguishing between physiological athletic heart and pathologic left ventricular hypertrophy has important implications for trained athletes or individuals with vigorous avocations. Pathologic left ventricular hypertrophy may be the basis for disqualification from intense competitive sport activities to reduce the risk of sudden death [28]. Alternatively, over diagnosis can lead to unnecessary restrictions, depriving athletes and/or exercise participants of the monetary and/or psychological benefits of vigorous aerobic sports. For the clinician faced with deciding whether this is structural heart disease or athlete's heart, an echocardiogram is very helpful [29]. Several considerations are important: (1) Left ventricular systolic and diastolic function is normal in the athlete. Remember, ejection fraction in a left ventricle with physiologic diastolic dilatation (LV cavity greater than $55 \mathrm{~mm}$ ) and a slow heart rate will be in the low normal range of 50-55 percent. (2) The left ventricular wall thickness is usually less than but not greater than $12 \mathrm{~mm}$. (3) The individual will give a history of training and competing in sports combining aerobic endurance and strength. (4) The individual will have a slow resting heart rate due to training-induced increased parasympathetic activity on the sinus node [27]. If the individual has a left ventricular end diastolic dimension of less than $45 \mathrm{~mm}$ and/or left ventricular wall thickness of greater than $15 \mathrm{~mm}$, pathologic left ventricular hypertrophy should be suspected $[27,30]$. Tests such as Doppler echocardiogram or cardiovascular magnetic resonance become important additional implements in the differential diagnosis in some patients. For example, about two percent of highly trained adult male athletes show increased left ventricular wall thickness of $13-15 \mathrm{~mm}$ which define a "gray zone" where extreme expressions of athlete heart and mild pathologic left ventricular hypertrophy overlap [31]. Such diagnostic ambiguity can often be resolved with noninvasive testing such as the assessment of diastolic filling with Doppler echocardiography.

Due to technical advances, cardiovascular magnetic resonance is now firmly established in research and clinical cardiovascular medicine. While echocardiography is a faster and more portable modality, cardiovascular magnetic resonance typically provides superior reproducible image quality. Both echocardiography and magnetic resonance are nonionizing technologies which in the modern climate 
of safety priority are favored over X-ray and gamma-ray modalities in the evaluation and long-term management of patients with left ventricular hypertrophy $[32,33]$. Changes in left ventricular mass can indicate disease progression or regression in hypertensive heart disease. Cardiovascular magnetic resonance is superior to echocardiology because of excellent reproducibility and because echocardiographic methods are dependent on symmetry on left ventricular shape [34]. Because of cost, echocardiology is more commonly used in clinical assessment of hypertensive patients. However, measuring changes in left ventricular mass using cardiovascular magnetic resonance has become a precise useful research tool when testing the benefit of pharmacologic treatment of hypertensive left ventricular hypertrophy [35]. While not the focus of this article, patients may present with hypertension, left ventricular hypertrophy on electrocardiogram, and increased left ventricular mass who have hypertrophic cardiomyopathy rather than hypertensive heart disease. Cardiovascular magnetic resonance imaging is useful to distinguish hypertensive and hypertrophic cardiomyopathy [36]. Recent studies indicate the presence of distinctive hypertrophic phenotypes detectable by means of multiparametric magnetic resonance imaging. In hypertension, impaired deformation follows the distribution of left ventricular wall stress. In contrast, hypertrophic cardiomyopathy is characterized by reduced global and regional deformation in association with fibrosis.

\section{Nonpharmacologic Strategies for Prevention and Regression of Pathologic Hypertrophy}

Effective, long-term antihypertensive therapy focused on the regression of left ventricular hypertrophy is indicated in these patients and requires specific nonpharmacologic and pharmacologic consideration and treatment. With the regression of left ventricular hypertrophy, diastolic function and coronary flow reserve usually improve, and risk of atrial fibrillation, congestive heart failure, and cardiovascular mortality decreases [37-42]. Goal of therapy is an arterial blood pressure of $130 / 80 \mathrm{~mm} \mathrm{Hg}$ or better. Left ventricular hypertrophy regression necessitates effective arterial blood pressure lowering 24 hours a day. In patients who appear to be at goal but fail to regress, 24-hour blood pressure ambulatory monitoring can be used to determine whether arterial pressure is, in fact, effectively controlled.

In addition to controlling arterial pressure, sodium restriction and weight loss independently facilitate the regression of left ventricular hypertrophy [43]. If the patient even loses 10 pounds, it enhances the antihypertensive effect of medications, and associated weight loss speeds up the potential for regression. Weight loss facilitating the regression of left ventricular hypertrophy has been accomplished through moderate calorie restriction in combination with regular low resistance exercise, such as walking 30 minutes a day [44]. Other interventions for successful treatment of hypertension to allow for the regression of left ventricular hypertrophy include minimizing use of nonsteroidal, antiinflammatory medications which inhibit synthesis of renal prostaglandin resulting in renal vasoconstriction, salt, and water retention and more difficult-to-control high blood pressure [24]. It is important to recognize excessive alcohol intake and limit it as a correctable factor for treatment of hypertension. It is also important to recognize sleep apnea and treat this condition, for sleep apnea can contribute to and cause hypertension, and treatment of sleep apnea has been shown to facilitate the regression of left ventricular hypertrophy [45].

\section{Pharmacologic Strategies for Prevention and Regression of Pathologic Hypertrophy}

Choice of antihypertensive agents may be important when treating a patient with hypertension and left ventricular hypertrophy. The classes of antihypertensive agents that most rapidly facilitate the regression of left ventricular hypertrophy are the angiotensin-converting enzyme inhibitors and the angiotensin II receptor blockers followed by calcium channel antagonists [46-49]. Angiotensin-converting enzyme inhibitors and angiotensin II receptor blockers are particularly effective because angiotensin II has been found to have a direct tropic effect of the pathogenesis of left ventricular hypertrophy $[42,50,51]$. Decrease in left ventricular mass may occur in as little as three to six months. The direct renin inhibitor aliskiren is also effective in facilitating the regression of left ventricular mass [52]. Thus far, the potential benefit of combining either an angiotensin II receptor blocker or an angiotensin-converting enzyme inhibitor with a direct renin inhibitor is under investigation. The initial trial combining an angiotensin II receptor blocker with a direct renin inhibitor did not show an additive benefit on the regression of left ventricular hypertrophy [52].

Angiotensin-converting enzyme inhibitors also appear to benefit patients with left ventricular hypertrophy because they improve coronary endothelial function by bradykininmediated release of nitric oxide and decrease myocardial oxygen consumption by nitric oxide inhibition of mitochondrial respiration [53]. When initiating angiotensinconverting enzyme inhibitors in patients, it is important to warn the patients of the life-threatening side effect of angioedema. This idiosyncratic side effect occurs in a small number of patients. When on angiotensin-converting enzyme therapy, patients should be warned if they start developing shortness of breath or swelling of the throat and tongue, they should stop the angiotensin-converting enzyme inhibitor and immediately go to the emergency room since this is a potentially life-threatening side effect. When starting an angiotensin-converting enzyme inhibitor, angiotensin II receptor blocker, or direct renin inhibitor, it is also important to screen for a change in renal function. A small number of patients who have unrecognized renal artery stenosis, whether it be large vessel or small vessel disease, will have a decrease in renal perfusion pressure because of the loss of intrarenal angiotensin II maintenance of renal perfusion balance between afferent and efferent arterioles. In these patients, serum creatinine will increase due to decreased glomerular filtration rate. This is easily 
detected by measuring a baseline serum creatinine and then checking serum creatinine again within a week. If serum creatinine increases by greater than $0.3 \mathrm{mg} / \mathrm{dL}$ from baseline, the angiotensin-converting enzyme inhibitor, angiotensin II receptor blocker, or direct renin inhibitor should be discontinued, and this reversible decrease in glomerular filtration rate will correct itself.

Thus far, the angiotensin-converting enzyme inhibitor and the angiotensin II receptor blocker trials suggest treating risk factors beyond high blood pressure and should, therefore, be used in hypertensive African American patients with left ventricular hypertrophy independent of blood pressure-lowering effects [54]. However, blood pressure control is paramount to reduce cardiovascular morbidity and mortality in these high-risk patients. Thus, other classes of antihypertensive agents such as diuretics and calcium channel blockers are usually needed in combination with an angiotensin-converting enzyme inhibitor or an angiotensin II receptor blocker to achieve goal blood pressure. Angiotensin-converting enzyme inhibitor cough has been found to be more common in African Americans than in whites. In contrast, angiotensin II receptor blockers have consistently been found to have a side-effect profile not greater than that of placebo. Therefore, an angiotensin II receptor blocker may become an important addition to the antihypertensive regimen of African Americans with left ventricular hypertrophy.

Besides facilitating the regression of left ventricular hypertrophy, calcium channel antagonists improve coronary blood flow in patients with left ventricular hypertrophy $[55,56]$. Longer-acting calcium channel antagonists are safe and appropriate for treatment of hypertensive patients with left ventricular hypertrophy. Choice of calcium channel antagonists should be dictated by the patient's resting heart rate and response to exercise. If a patient has a hyperadrenergic response to exercise, verapamil, or diltiazem are appropriate choices for symptomatic relief because these calcium channel antagonists attenuate both the heart rate and blood pressure response to exercise. In contrast, traditional beta-adrenergic receptor blocking agents only attenuate the heart rate response to exercise and actually can (due to unopposed alpha constriction) cause an accentuated systolic blood pressure elevation during exercise. In contrast, carvedilol and labetalol which have alpha-blocking properties attenuate both heart rate and exercise response similar to verapamil and diltiazem, thus, providing symptomatic relief of symptoms of dyspnea or angina and also these agents facilitate a slower heart rate to allow for better diastolic filling of the ventricle. In older patients who have conduction system disease and slower heart rates, dihydropyridines are beneficial, and the dihydropyridine which best seems to facilitate the regression of left ventricular hypertrophy is amlodipine [49].

Several lines of evidence suggest that combining an angiotensin-converting enzyme inhibitor or an angiotensin II receptor blocker with amlodipine may have additive effects, not only in blood pressure regulation, but to facilitate the regression of left ventricular hypertrophy and also decrease mortality [49]. Peripheral alpha-adrenergic blocking agents, thiazide-type diuretics, and even traditional beta-adrenergic receptor blocking agents also allow for left ventricular hypertrophy regression over time $[24,46]$. Centrally acting alpha agonists have not been carefully studied for their effects on left ventricular hypertrophy regression. Direct vasodilators, such as minoxidil and hydralazine, which further stimulate renin-angiotensin-aldosterone system and sympathetic nervous system activity, although effective in controlling blood pressure, are ineffective with regard to the regression of left ventricular hypertrophy [24].

Current treatment recommendations for hypertensive patients with left ventricular hypertrophy are based on blood pressure lowering and do not specifically favor selection of a particular beta-blocker over another except to avoid beta-blockers with intrinsic sympathomimetic activity which may increase left ventricular mass and increase the risk of sudden death [24]. However, recent trials emphasize the class heterogeneity that exists for beta-blockers and provide a strong basis for preferred use of carvedilol in these high-risk patients. Carvedilol is a unique molecule that combines the properties of a nonspecific beta-blocker and a specific alpha-1-blocker in a ratio of $2: 3$. Carvedilol also possesses antioxidant properties. Several studies have shown that carvedilol significantly reduces left ventricular mass and myocardial fibrosis in both animals and humans $[57,58]$. In patients with hypertensive left ventricular hypertrophy and decreased coronary flow reserve, carvedilol treatment compared to metoprolol treatment over six months showed a greater decrease in left ventricular mass index and a greater recovery in coronary flow reserve [59].

Patients with hypertensive left ventricular hypertrophy are at an increased risk of progressing to left ventricular systolic dysfunction heart failure [60]. Studies have shown that angiotensin-converting enzyme inhibitors delay the development of congestive heart failure [51]. Animal studies indicate a role of oxidate stress causing myocyte apoptosis in the transition of hypertrophy to systolic heart failure [61]. Carvedilol with its complete sympathetic blockade and antioxidant properties may be an appropriate choice for the prevention of heart failure in these patients. This concept is reinforced by the beneficial effects of carvedilol for the reduction in morbidity and mortality in patients after myocardial infarction and with systolic congestive heart failure $[62,63]$.

Left ventricular hypertrophy and dyslipidemia are strong and independent predictors of cardiovascular morbidity and mortality with hypertension. Traditional beta-blockers have been shown to worsen insulin resistance, facilitate weight gain, and increase triglycerides [64]. Carvedilol in hypertensive patients with diabetes has been found to have a neutral effect on insulin resistance, weight, and triglycerides [64]. This favorable metabolic profile also suggests that carvedilol is a better choice compared to traditional beta-blockers in these high-risk patients. Similarly, other antihypertensive agents indicated for treatment of hypertensive patients with left ventricular hypertrophy, including angiotensinconverting enzyme inhibitors, angiotensin II receptor blockers, calcium channel blockers, and central sympatholytic 
agents are lipid neutral. In contrast, peripheral alphaadrenergic blocking agents have been shown to increase HDL cholesterol as well as decrease total and LDL cholesterol [24]. Thiazide-type diuretics and to some degree loop diuretics increase total cholesterol. The thiazide-type diuretics at dosages of 6.25 or $12.5 \mathrm{mg}$ daily have only small effects on total cholesterol. Of note, indapamide is lipid neutral and thus has a somewhat different pharmacologic effect on lipids compared to the other diuretics [24].

Diuretics in low dosage enhance the antihypertensive effects of most antihypertensive medications. Older patients with stiff vessels usually have a volume-sensitive hypertension, and a low dose of a thiazide-type diuretic can be very effective therapy. However, when a patient's creatinine clearance is less than $50 \mathrm{~mL} / \mathrm{min}$, most thiazide-type diuretics are less effective for volume control and antihypertensive therapy. In these patients, alternatives would be chlorthalidone or indapamide which are effective despite decreased renal function. Loop diuretics can also be used in patients with decreased renal function, but to achieve antihypertensive responses, a loop diuretic, such as furosemide has to be given twice daily. In patients with resistant hypertension, thiazide-type diuretic is very important component of the pharmacologic antihypertensive therapy. In the patient with hypertension and left ventricular hypertrophy requiring diuretic therapy, it is important to keep the potassium between 4 and $5 \mathrm{mEq} / \mathrm{L}$ and magnesium at $2 \mathrm{mEq} / \mathrm{L}$ or greater to decrease the risk of ventricular arrhythmias [65, 66]. When extracellular potassium is less than $4 \mathrm{mEq} / \mathrm{L}$ or greater than $5 \mathrm{mEq} / \mathrm{L}$, atrial and ventricular cells are depolarized and more prone to atrial and ventricular arrhythmias. Extracellular hypomagnesemia has also been shown to depolarize atrial and ventricular muscle cells making them more prone to atrial and ventricular arrhythmias [67]. Serum potassium and magnesium levels reflect extracellular potassium and magnesium and, thus, are a sensitive guide to replacement therapy. Left ventricular hypertrophy is a substrate for ventricular arrhythmias because left ventricular hypertrophy prolongs the QT interval. Left ventricular hypertrophy in the setting of low potassium and/or low magnesium increases the risk of ventricular arrhythmias and even sudden death.

Evidence indicates that the aldosterone receptor blockers, spironolactone, or eplerenone are effective in facilitating the regression of left ventricular hypertrophy [68]. High aldosterone levels in experimental animals have been found to increase the fibrosis in the cardiac interstitial space, and fibrosis is part of the process of pathologic left ventricular hypertrophy. In experimental animals with left ventricular hypertrophy, the use of spironolactone or eplerenone decreases the development of fibrosis in pathologic left ventricular hypertrophy and even seems to facilitate its regression. If a thiazide-type diuretic is required, concomitant use of spironolactone or eplerenone is useful in conserving potassium and magnesium as well as facilitating the regression of left ventricular hypertrophy. Of note, betaadrenergic receptor blocking agents, angiotensin-converting enzyme inhibitors, and angiotensin II receptor blockers tend to normalize the QT interval in patients with left ventricular hypertrophy. Using the above pharmacologic agents, it is important to not only achieve goal of blood pressure of $130 / 80 \mathrm{~mm} \mathrm{Hg}$ or better, but also to attenuate blood pressure variability. Choosing antihypertensive agents with longer half lives has a smoothing effect on blood pressure and may explain the mortality benefits of longer-acting angiotensin-converting enzyme inhibitors, angiotensin II receptor blockers, amlodipine, and chlorthalidone [69]. Angiotensin-converting enzyme inhibitors, angiotensin II receptor blockers, and dihydropyridine calcium-channel blockers may also be effective in preventing or facilitating the regression of left ventricular hypertrophy because these medications reduce reflected pressure waves thus lowering central (aortic) blood pressure [70]. A 3-4 mm Hg lower central (aortic) blood pressure over time should result in less left ventricular hypertrophy.

\section{Future Novel Approaches to the Treatment of Left Ventricular Hypertrophy in Patients with Hypertension}

A future potential therapeutic concept for the treatment of hypertension and to facilitate the regression of left ventricular hypertrophy is inhibitors of 3-hydroxy-3-methylglutaryl coenzyme A reductase (statins). Statins have been shown to facilitate the regression of left ventricular hypertrophy in various animal models of pathological myocardial growth $[71,72]$. In addition to the reduction of left ventricular mass, statins have been shown to reduce myocardial fibrosis, increase capillary density network, and attenuate electrical instability of the hypertrophied heart. Most importantly, statins improved systolic and diastolic left ventricular function and even decreased mortality. The mechanisms of these beneficial effects are still under investigation. As statins lower cholesterol, endothelial function improves and systolic blood decreases by $2-4 \mathrm{~mm} \mathrm{Hg}$. The inhibition of hypertrophic growth by statins is only partly achieved by the reduction of hemodynamic overload. Direct mechanisms such as the inhibition of neurohumoral activation in the myocardial tissue, attenuated production of growth factors and markers of inflammation, and the reduction of oxidative stress also seem to participate. Thus, statins appear to exert biological effects other than their cholesterol-lowering actions. The additional protective effect of statins is associated with the inhibition of expression and activation of small guanosin triphosphate-binding proteins such as Ras and Rho which control the intensity of oxidative stress, the production and availability of nitric oxide, and the expression of genes involved in myocardial growth. The data from these carefully done animal studies document novel effects of statins on left ventricular hypertrophy and open a potential new therapeutic strategy for patients with cardiac hypertrophy. Clinical trials to test statins benefit in patients at risk with left ventricular hypertrophy are in progress.

Another potential therapeutic target for the treatment of hypertension and to facilitate the regression of left ventricular hypertrophy is the renal sympathetic efferent and afferent nerves, which lie within and immediately adjacent 
to the wall of the renal arteries, and contribute to the maintenance of hypertension [73]. Because the causative factors of hypertension change over time, the denervation of both efferent and afferent renal sympathetic nerves should result in long-term attenuation of hypertension. The importance of the renal nerves in the hypertensive patient has now been defined with the novel development of percutaneous, minimally invasive renal denervation from within the renal artery using radiofrequency energy as a therapeutic strategy. Studies, thus far, show that catheterbased renal denervation in patients with resistant essential hypertension lowers systolic blood pressure $27 \mathrm{~mm} \mathrm{Hg}$ by 12 months with the estimated glomerular filtration rate remaining stable [74]. The decrease in arterial pressure after renal denervation is associated with decreased peripheral sympathetic nervous system activity suggesting that the kidney is a source of significant sympathetic outflow via afferent renal nerve activity. Patients whose hypertension is mediated by important sympathetic nervous systemrenal factors represent a new potential therapeutic target for, not only treatment of resistant hypertension, but also left ventricular hypertrophy. Since increased central sympathetic outflow is believed to contribute to left ventricular hypertrophy, the reduction of central sympathetic outflow following renal denervation may facilitate the regression of left ventricular hypertrophy. If proved true in prospective studies, this therapy may have unique value in reversing left ventricular hypertrophy. This concept is supported, thus far, in a recent case report of a 59-year-old patient with longstanding essential hypertension resistant to multidrug treatment [75]. After renal denervation, a gradual reduction of systolic blood pressure of $30 \mathrm{~mm} \mathrm{Hg}$ over 12 months was accompanied by an eight percent decrease in left ventricular mass as measured by cardiovascular magnetic resonance.

\section{Conclusions}

Hypertensive left ventricular hypertrophy is a pathologic response resulting in diastolic dysfunction and decreased coronary flow reserve. Left ventricular hypertrophy increases the risk for atrial fibrillation, diastolic heart failure, systolic heart failure, and sudden death in patients with hypertension. An echocardiogram and cardiovascular magnetic resonance are useful tools to access left ventricular mass. Controlling arterial pressure, sodium restriction, and weight loss independently facilitate the regression of left ventricular hypertrophy. Angiotensin-converting enzyme inhibitors or angiotensin II receptor blockers followed by calcium channel antagonists most rapidly facilitate the regression of left ventricular hypertrophy. Increasing evidence indicates that aldosterone receptor blockers are effective in facilitating the regression of left ventricular hypertrophy. Chlorthalidone appears to be the most effective thiazide-type diuretic for treatment of hypertension. Carvedilol appears to be the beta-blocker of choice in patients with hypertensive left ventricular hypertrophy. Future novel approaches to the treatment of left ventricular hypertrophy in patients with hypertension are to facilitate regression with statins and to attenuate the hypertension using cather-based renal sympathetic denervation.

\section{Conflicts of Interest}

The authors declare that there are no conflict of interests relevant to this article.

\section{Acknowledgment}

Special thanks to Marcey R. Ervin and Sandy Sledge for preparation of the manuscript and literature search for references.

\section{References}

[1] A. Sagie, E. J. Benjamin, M. Galderisi et al., "Echocardiographic assessment of left ventricular structure and diastolic filling in elderly subjects with borderline isolated systolic hypertension (the Framingham Heart Study)," American Journal of Cardiology, vol. 72, no. 9, pp. 662-665, 1993.

[2] W. S. Post, M. G. Larson, and D. Levy, "Impact of left ventricular structure on the incidence of hypertension: the Framingham Heart Study," Circulation, vol. 90, no. 1, pp. 179185, 1994.

[3] P. Anversa, E. Puntillo, G. Olivetti et al., "Cellular and mechanical adaptations in cardiac hypertrophy and its reversal," Cardiovascular Reviews and Reports, vol. 11, no. 12, pp. 34-41, 1990.

[4] D. B. Johnson and L. J. Dell'Italia, "Cardiac hypertrophy and failure in hypertension," Current Opinion in Nephrology and Hypertension, vol. 5, no. 2, pp. 186-191, 1996.

[5] T. E. Simpson, H. M. Dansky, and P. M. Buttrick, "Molecular genetic mechanisms of cardiac hypertrophy," Cardiovascular Risk Factors, vol. 5, no. 2, pp. 93-108, 1995.

[6] M. Moser, "Initial workup of the hypertensive patient," in Hypertensive Primer: The Essentials of High Blood Pressure, J. L. Izzo, H. R. Black, and T. L. Goodfriend, Eds., pp. 221-223, Williams and Wilkins, Philadelphia, Pa, USA, 1998.

[7] W. B. Kannel, "Prevalence and natural history of electrocardiographic left ventricular hypertrophy," American Journal of Medicine, vol. 75, no. 3 A, pp. 4-11, 1983.

[8] P. N. Casale, R. B. Devereux, and M. Milner, "Value of echocardiographic measurement of left ventricular mass in predicting cardiovascular morbid events in hypertensive men," Annals of Internal Medicine, vol. 105, no. 2, pp. 173-178, 1986.

[9] Y. Sugishita, K. Iida, K. Fujieda, and K. Yukisada, "Decreased adrenergic response in hypertensive patients without left ventricular hypertrophy," Clinical Cardiology, vol. 17, no. 2, pp. 71-76, 1994.

[10] R. E. Katholi, M. R. Ervin, and G. Ginsburg, "Hypertensive heart disease syndrome in women in a cardiology practice," Comprehensive Therapy, vol. 23, no. 8, pp. 508-513, 1997.

[11] Y. Liao, R. S. Cooper, G. A. Mensah, and D. L. McGee, "Left ventricular hypertrophy has a greater impact on survival in women than in men," Circulation, vol. 92, no. 4, pp. 805-810, 1995.

[12] S. Wassertheil-Smoller, B. Psaty, P. Greenland et al., "Association between cardiovascular outcomes and antihypertensive drug treatment in older women," Journal of the American Medical Association, vol. 292, no. 23, pp. 2849-2859, 2004.

[13] J. S. Mogil, W. F. Sternberg, B. Kest, P. Marek, and J. C. Liebeskind, "Sex differences in the antagonism of swim 
stress-induced analgesia: effects of gonadectomy and estrogen replacement," Pain, vol. 53, no. 1, pp. 17-25, 1993.

[14] G. M. C. Rosano, N. S. Peters, D. Lefroy et al., "17-betaestradiol therapy lessens angina in postmenopausal women with syndrome X," Journal of the American College of Cardiology, vol. 28, no. 6, pp. 1500-1505, 1996.

[15] G. Mulé, E. Nardi, S. Cottone et al., "Impact of the metabolic syndrome on total arterial compliance in essential hypertension patients," Journal of the Cardiometabolic Syndrome, vol. 2, no. 2, pp. 84-90, 2007.

[16] P. Verdecchia, G. Schillaci, M. Guerrieri et al., "Circadian blood pressure changes and left ventricular hypertrophy in essential hypertension," Circulation, vol. 81, no. 2, pp. 528 536, 1990.

[17] A. Sharp, R. Tapp, D. P. Francis et al., "Ethnicity and left ventricular diastolic function in hypertension an ASCOT (AngloScandinavian Cardiac Outcomes Trial) substudy," Journal of the American College of Cardiology, vol. 52, no. 12, pp. 10151021, 2008.

[18] A. M. Grandi, R. Broggi, S. Colombo et al., "Left ventricular changes in isolated office hypertension: a blood pressurematched comparison with normotension and sustained hypertension," Archives of Internal Medicine, vol. 161, no. 22, pp. 2677-2681, 2001.

[19] A. Losito, R. M. Fagugli, I. Zampi et al., "Comparison of target organ damage in renovascular and essential hypertension," American Journal of Hypertension, vol. 9, no. 11, pp. 10621067, 1996.

[20] S. Wu, N. Polavarapu, and G. A. Stouffer, "Left ventricular hypertrophy in patients with renal artery stenosis," American Journal of the Medical Sciences, vol. 332, no. 6, pp. 334-338, 2006.

[21] G. P. Rossi, A. Sacchetto, E. Pavan et al., "Remodeling of the left ventricle in primary aldosteronism due to Conn's adenoma," Circulation, vol. 95, no. 6, pp. 1471-1478, 1997.

[22] W. H. Frishman, "Diagnosis and treatment of systolic heart failure in the elderly," American Journal of Geriatric Cardiology, vol. 7, no. 6, pp. 10-55, 1998.

[23] R. N. Foley, P. S. Parfrey, J. D. Harnett, G. M. Kent, D. C. Murray, and P. E. Barre, "Impact of hypertension on cardiomyopathy, morbidity and mortality in end-stage renal disease," Kidney International, vol. 49, no. 5, pp. 1379-1385, 1996.

[24] R. E. Katholi, "Left ventricular hypertrophy: strategies for prevention and regression," Midwest Health, vol. 2, pp. 26-32, 2000.

[25] G. W. Ching, J. A. Franklyn, T. J. Stallard, J. Daykin, M. C. Sheppard, and M. D. Gammage, "Cardiac hypertrophy as a result of long-term thyroxine therapy and thyrotoxicosis," Heart, vol. 75, no. 4, pp. 363-368, 1996.

[26] J. P. Singh, J. Johnston, P. Sleight, R. Bird, K. Ryder, and G. Hart, "Left ventricular hypertrophy in hypertensive patients is associated with abnormal rate adaptation of QT interval," Journal of the American College of Cardiology, vol. 29, no. 4, pp. 778-784, 1997.

[27] B. J. Maron, A. Pelliccia, and P. Spirito, "Cardiac disease in young trained athletes: insights into methods for distinguishing athlete's heart from structural heart disease, with particular emphasis on hypertrophic cardiomyopathy," Circulation, vol. 91, no. 5, pp. 1596-1601, 1995.

[28] B. J. Maron, "Sudden death in young athletes," New England Journal of Medicine, vol. 349, no. 11, pp. 1064-1075, 2003.
[29] R. B. Devereux and N. Reichek, "Echocardiographic determination of left ventricular mass in man. Anatomic validation of the method," Circulation, vol. 55, no. 4, pp. 613-618, 1977.

[30] P. S. Douglas, M. L. O’Toole, S. E. Katz, G. S. Ginsburg, W. D. B. Hiller, and R. H. Laird, "Left ventricular hypertrophy in athletes," American Journal of Cardiology, vol. 80, no. 10, pp. 1384-1388, 1997.

[31] A. Pelliccia, F. Culasso, F. M. Di Paolo, and B. J. Maron, "Physiologic left ventricular cavity dilatation in elite athletes," Annals of Internal Medicine, vol. 130, no. 1, pp. 23-31, 1999.

[32] N. Reichek, J. Helak, and T. Plappert, "Anatomic validation of left ventricular mass estimates from clinical two-dimensional echocardiography: initial results," Circulation, vol. 67, no. 2, pp. 348-352, 1983.

[33] S. G. Myerson, N. G. Bellenger, and D. J. Pennell, "Assessment of left ventricular mass by cardiovascular magnetic resonance," Hypertension, vol. 39, no. 3, pp. 750-755, 2002.

[34] J. C. C. Moon, N. G. Fisher, W. J. McKenna, and D. J. Pennell, "Detection of apical hypertrophic cardiomyopathy by cardiovascular magnetic resonance in patients with nondiagnostic echocardiography," Heart, vol. 90, no. 6, pp. 645649, 2004.

[35] I. Olivotto, B. J. Maron, E. Appelbaum et al., "Spectrum and clinical significance of systolic function and myocardial fibrosis assessed by cardiovascular magnetic resonance in hypertrophic cardiomyopathy," American Journal of Cardiology, vol. 106, no. 2, pp. 261-267, 2010.

[36] V. O. Puntmann, C. Jahnke, R. Gebker et al., "Usefulness of magnetic resonance imaging to distinguish hypertensive and hypertrophic cardiomyopathy," American Journal of Cardiology, vol. 106, no. 7, pp. 1016-1022, 2010.

[37] M. L. Muiesan, M. Salvetti, D. Rizzoni, M. Castellano, F. Donato, and E. Agabiti-Rosei, "Association of change in left ventricular mass with prognosis during long-term antihypertensive treatment," Journal of Hypertension, vol. 13, no. 10, pp. 1091-1095, 1995.

[38] R. B. Devereux, E. Agabiti-Rosei, B. Dahlof et al., "Regression of left ventricular hypertrophy as a surrogate end-point for morbid events in hypertension treatment trials," Journal of Hypertension, Supplement, vol. 14, no. 2, pp. S95-S102, 1996.

[39] R. B. Devereux, K. Wachtell, E. Gerdts et al., "Prognostic significance of left ventricular mass change during treatment of hypertension," Journal of the American Medical Association, vol. 292, no. 19, pp. 2350-2356, 2004.

[40] P. M. Okin, R. B. Devereux, S. Jern et al., "Regression of electrocardiographic left ventricular hypertrophy during antihypertensive treatment and the prediction of major cardiovascular events," Journal of the American Medical Association, vol. 292, no. 19, pp. 2343-2349, 2004.

[41] P. M. Okin, R. B. Devereux, K. E. Harris et al., "Regression of electrocardiographic left ventricular hypertrophy is associated with less hospitalization for heart failure in hypertensive patients," Annals of Internal Medicine, vol. 147, no. 5, pp. 311319, 2007.

[42] K. Wachtell, P. M. Okin, M. H. Olsen et al., "Regression of electrocardiographic left ventricular hypertrophy during antihypertensive therapy and reduction in sudden cardiac death: the LIFE study," Circulation, vol. 116, no. 7, pp. 700705, 2007.

[43] L. A. Ferrara, G. De Simone, F. Pasanisi, and M. Mancini, "Left ventricular mass reduction during salt depletion in arterial hypertension," Hypertension, vol. 6, no. 5, pp. 755-759, 1984. 
[44] B. Arroll and R. Beaglehole, "Does physical activity lower blood pressure: a critical review of the clinical trials," Journal of Clinical Epidemiology, vol. 45, no. 5, pp. 439-447, 1992.

[45] H. F. Becker, A. Jerrentrup, T. Ploch et al., "Effect of nasal continuous positive airway pressure treatment on blood pressure in patients with obstructive sleep apnea," Circulation, vol. 107, no. 1, pp. 68-73, 2003.

[46] R. E. Schmieder, P. Martus, and A. Klingbeil, "Reversal of left ventricular hypertrophy in essential hypertension: a metaanalysis of randomized double-blind studies," Journal of the American Medical Association, vol. 275, no. 19, pp. 1507-1513, 1996.

[47] P. R. Liebson, G. A. Grandits, S. Dianzumba et al., "Comparison of five antihypertensive monotherapies and placebo for change in left ventricular mass in patients receiving nutritional-hygienic therapy in the treatment of mild hypertension study (TOMHS)," Circulation, vol. 91, no. 3, pp. 698706, 1995.

[48] J. S. Gottdiener, D. J. Reda, B. M. Massie, B. J. Materson, D. W. Williams, and R. J. Anderson, "Effect of single-drug therapy on reduction of left ventricular mass in mild to moderate hypertension: comparison of six antihypertensive agents," Circulation, vol. 95, no. 8, pp. 2007-2014, 1997.

[49] R. J. Tapp, A. Sharp, A. V. Stanton et al., "Differential effects of antihypertensive treatment on left ventricular diastolic function: an ASCOT (Anglo-Scandinavian Cardiac Outcomes Trial) substudy," Journal of the American College of Cardiology, vol. 55, no. 17, pp. 1875-1881, 2010.

[50] M. Iriarte, R. Caso, N. Murga et al., "Enalapril-induced regression of hypertensive left ventricular hypertrophy, regional ischemia, and microvascular angina," American Journal of Cardiology, vol. 75, no. 12, pp. 850-852, 1995.

[51] J. Mathew, P. Sleight, E. Lonn et al., "Reduction of cardiovascular risk by regression of electrocardiographic markers of left ventricular hypertrophy by the angiotensin-converting enzyme inhibitor ramipril," Circulation, vol. 104, no. 14, pp. 1615-1621, 2001.

[52] S. D. Solomon, E. Appelbaum, W. J. Manning et al., "Effect of the direct renin inhibitor aliskiren, the angiotensin receptor blocker losartan, or both on left ventricular mass in patients with hypertension and left ventricular hypertrophy," Circulation, vol. 119, no. 4, pp. 530-537, 2009.

[53] X. Zhang, Y. W. Xie, A. Nasjletti, X. Xu, M. S. Wolin, and T. H. Hintze, "ACE inhibitors promote nitric oxide accumulation to modulate myocardial oxygen consumption," Circulation, vol. 95, no. 1, pp. 176-182, 1997.

[54] S. E. Nissen, E. M. Tuzcu, P. Libby et al., "Effect of antihypertensive agents on cardiovascular events in patients with coronary disease and normal blood pressure. The CAMELOT study: a randomized controlled trial," Journal of the American Medical Association, vol. 292, no. 18, pp. 2217-2226, 2004.

[55] W. H. Frishman, A. E. Skolnick, and J. A. Strom, "Effects of calcium entry blockade on hypertension-induced left ventricular hypertrophy," Circulation, vol. 80, no. 6, pp. IV151-IV161, 1989.

[56] M. Vogt and B. E. Strauer, "Response of hypertensive left ventricular hypertrophy and coronary microvascular disease to calcium antagonists," American Journal of Cardiology, vol. 76, no. 13, pp. 24D-30D, 1995.

[57] F. C. Barone, W. G. Campbell Jr., A. H. Nelson, and G. Z. Feuerstein, "Carvedilol prevents severe hypertensive cardiomyopathy and remodeling," Journal of Hypertension, vol. 16, no. 6, pp. 871-884, 1998.
[58] L. Hansson and A. Himmelmann, "Carvedilol in the treatment of hypertension - a review of the clinical data base," Scandinavian Cardiovascular Journal, Supplement, vol. 32, no. 47, pp. 67-80, 1998.

[59] H. Xiaozhen, Z. Yun, Z. Mei, and S. Yu, "Effect of carvedilol on coronary flow reserve in patients with hypertensive leftventricular hypertrophy," Blood Pressure, vol. 19, no. 1, pp. 4047, 2010

[60] D. Levy, M. G. Larson, R. S. Vasan, W. B. Kannel, and K. K. L. Ho, "The progression from hypertension to congestive heart failure," Journal of the American Medical Association, vol. 275, no. 20, pp. 1557-1562, 1996.

[61] A. K. Dhalla, M. F. Hill, and P. K. Singal, "Role of oxidative stress in transition of hypertrophy to heart failure," Journal of the American College of Cardiology, vol. 28, no. 2, pp. 506-514, 1996.

[62] H. J. Dargie, "Effect of carvedilol on outcome after myocardial infarction in patients with left-ventricular dysfunction: the CAPRICORN randomised trial," Lancet, vol. 357, no. 9266, pp. 1385-1390, 2001.

[63] M. Packer, M. R. Bristow, J. N. Cohn et al., "The effect of carvedilol on morbidity and mortality in patients with chronic heart failure," New England Journal of Medicine, vol. 334, no. 21, pp. 1349-1355, 1996.

[64] G. L. Bakris, V. Fonseca, R. E. Katholi et al., "Metabolic effects of carvedilol vs metoprolol in patients with type 2 diabetes mellitus and hypertension: a randomized controlled trial," Journal of the American Medical Association, vol. 292, no. 18, pp. 2227-2236, 2004.

[65] D. S. Siscovick, T. E. Raghunathan, B. M. Psaty et al., "Diuretic therapy for hypertension and the risk of primary cardiac arrest," New England Journal of Medicine, vol. 330, no. 26, pp. 1852-1857, 1994.

[66] A. Bayes-Genis, J. Guindo, X. Vinolas et al., "Cardiac arrhythmias and left ventricular hypertrophy in systemic hypertension and their influences on prognosis," American Journal of Cardiology, vol. 76, no. 13, pp. 54D-59D, 1995.

[67] W. T. Woods, R. E. Katholi, F. Urthaler, and T. N. James, "Electrophysiological effects of magnesium on cells in the canine sinus node and false tendon," Circulation Research, vol. 44 , no. 2, pp. 182-188, 1979.

[68] V. K. Raman, Y. A. Lee, and K. Lindpaintner, "The cardiac renin-angiotensin-aldosterone system and hypertensive cardiac hypertrophy," American Journal of Cardiology, vol. 76, no. 13, pp. 18D-23D, 1995.

[69] M. Epstein and G. Bakris, "Newer approaches to antihypertensive therapy. Use of fixed-dose combination therapy," Archives of Internal Medicine, vol. 156, no. 17, pp. 1969-1978, 1996.

[70] B. Williams and P. S. Lacy, "Impact of heart rate on central aortic pressures and hemodynamics: analysis from the CAFE (Conduit Artery Function Evaluation) study: CAFE-Heart Rate," Journal of the American College of Cardiology, vol. 54, no. 8, pp. 705-713, 2009.

[71] C. Indolfi, E. Di Lorenzo, C. Perrino et al., "Hydroxymethylglutaryl coenzyme a reductase inhibitor simvastatin prevents cardiac hypertrophy induced by pressure overload and inhibits p21ras activation," Circulation, vol. 106, no. 16, pp. 2118$2124,2002$.

[72] F. Simko, "Statins: a perspective for left ventricular hypertrophy treatment," European Journal of Clinical Investigation, vol. 37, no. 9, pp. 681-691, 2007.

[73] R. E. Katholi, K. J. Rocha-Singh, N. J. Goswami, and P. A. Sobotka, "Renal nerves in the maintenance of hypertension: 
a potential therapeutic target," Current Hypertension Reports, vol. 12, pp. 196-204, 2010.

[74] H. Krum, M. Schlaich, R. Whitbourn et al., "Catheter-based renal sympathetic denervation for resistant hypertension: a multicentre safety and proof-of-principle cohort study," The Lancet, vol. 373, no. 9671, pp. 1275-1281, 2009.

[75] M. P. Schlaich, P. A. Sobotka, H. Krum, E. Lambert, and M. D. Esler, "Renal sympathetic-nerve ablation for uncontrolled hypertension," New England Journal of Medicine, vol. 361, no. 9, pp. 932-934, 2009. 


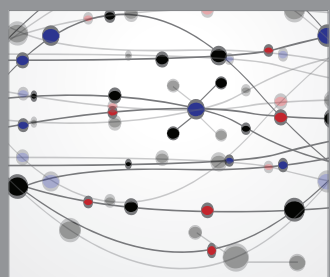

The Scientific World Journal
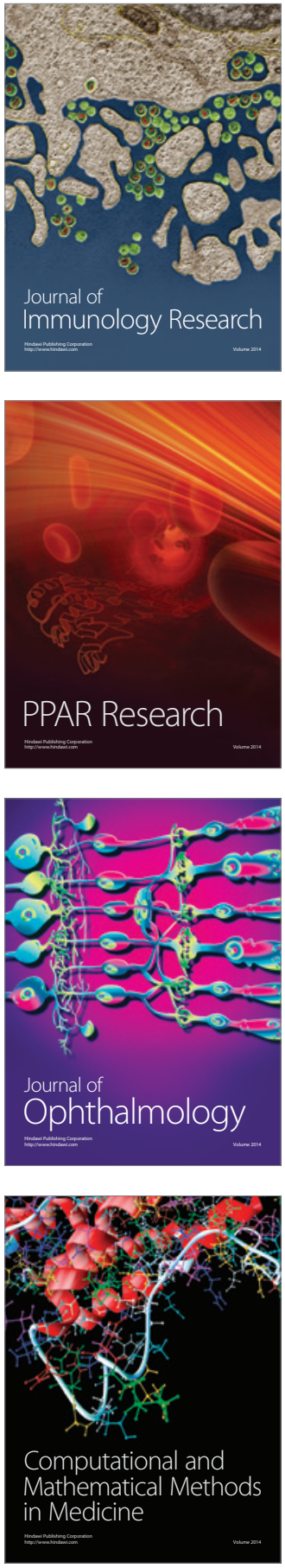

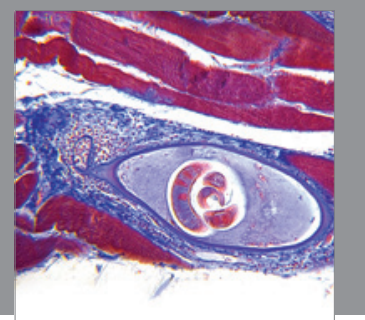

Gastroenterology

Research and Practice
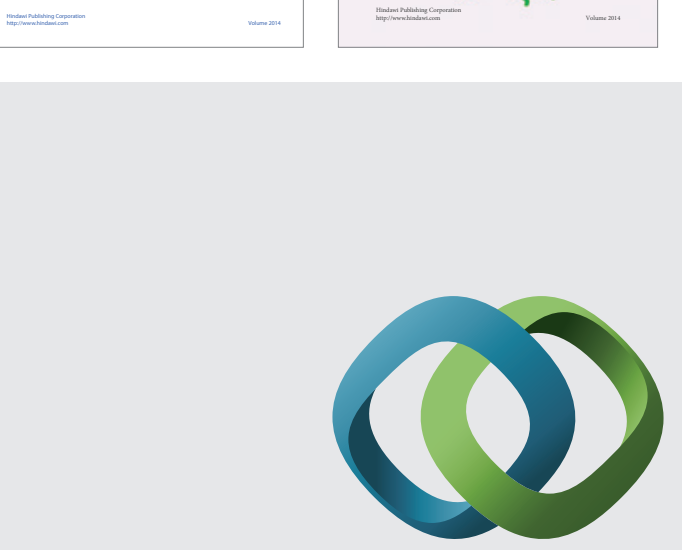

\section{Hindawi}

Submit your manuscripts at

http://www.hindawi.com
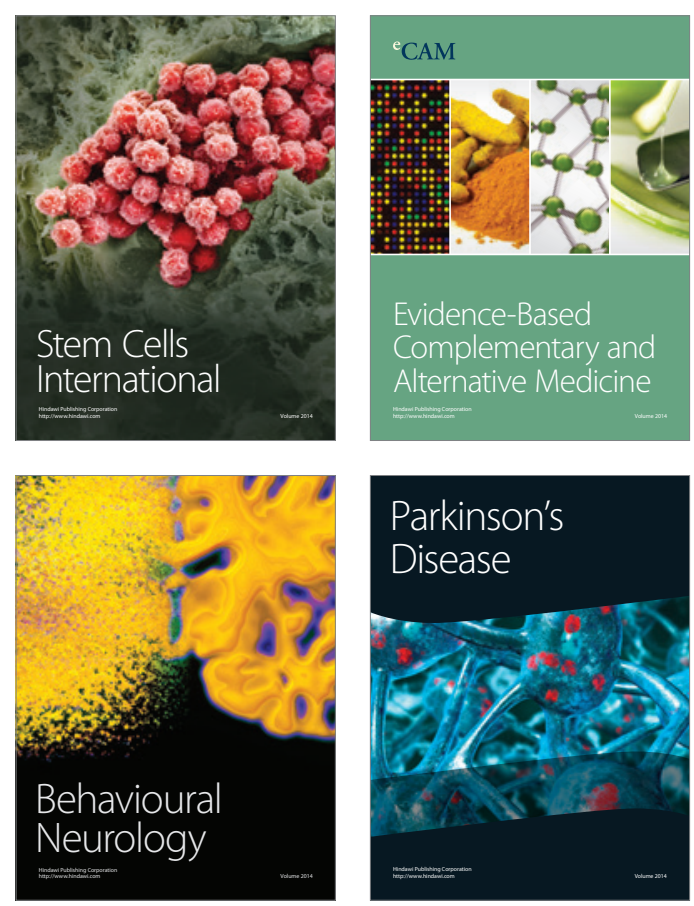

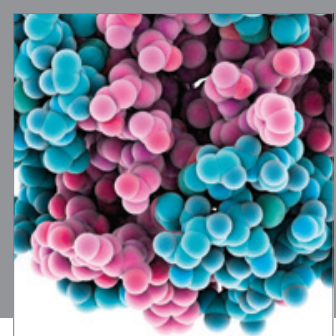

Journal of
Diabetes Research

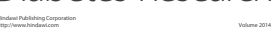

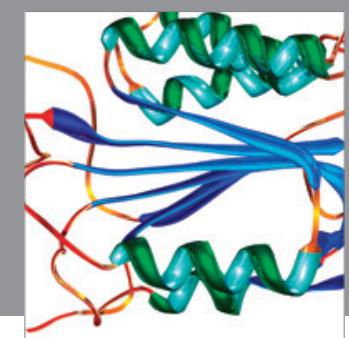

Disease Markers
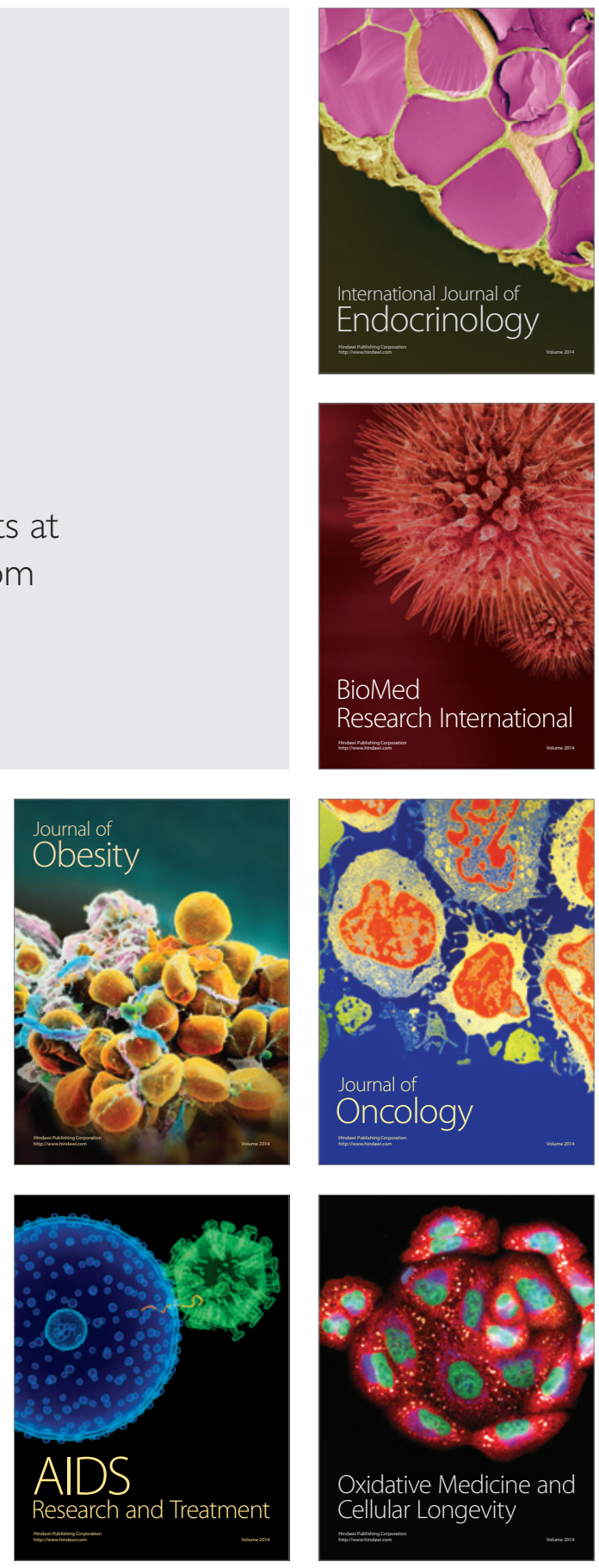